NASA Contractor Report 195342

$$
\begin{gathered}
1 N-64 \\
22319 \\
250
\end{gathered}
$$

\title{
Efficient and Accurate Explicit Integration Algorithms With Application to Viscoplastic Models
}

Vinod K. Arya

University of Toledo

Toledo, Ohio
N95-11464

Unclas VISCOPLASTIC MODELS Final Report (roledo Univ.) $25 \mathrm{p}$

G3/64 0022319

August 1994

Prepared for

Lewis Research Center

Under Cooperative Agreement NCC3-120

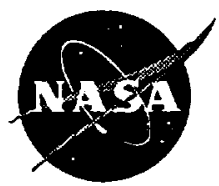

National Aeronautics and

Space Administration 


\title{
EFFICIENT AND ACCURATE EXPLICIT INTEGRATION \\ ALGORITHMS WITH APPLICATION TO \\ VISCOPLASTIC MODELS
}

\author{
Vinod K. Arya* \\ University of Toledo \\ Toledo, Ohio
}

\section{SUMMARY}

Several explicit integration algorithms with self-adaptive time integration strategies are developed and investigated for efficiency and accuracy. These algorithms involve the Runge-Kutta second order, the lower Runge-Kutta method of orders one and two, and the exponential integration method. The algorithms are applied to viscoplastic models put forth by Freed and Verrilli and Bodner and Partom for thermal/mechanical loadings (including tensile, relaxation, and cyclic loadings).

The large amount of computations performed showed that, for comparable accuracy, the efficiency of an integration algorithm depends significantly on the type of application (loading). However, in general, for the aforementioned loadings and viscoplastic models, the exponential integration algorithm with the proposed self-adaptive time integration strategy worked more (or comparably) efficiently and accurately than the other integration algorithms. Using this strategy for integrating viscoplastic models may lead to considerable saving in computer time (better efficiency) without adversely affecting the accuracy of the results. This conclusion should encourage the utilization of viscoplastic models in the stress analysis and design of structural components.

\section{INTRODUCTION}

The need for an accurate assessment of the time-dependent, inelastic response of structural components has led to the development of numerous constitutive material models, called viscoplastic models. Accurate assessment of inelastic response is an essential input for predicting the service life of components. Viscoplastic models incorporate all aspects of inelastic deformation, including plasticity, creep, and relaxation. These models do not endeavor to artificially split the inelastic strain into plastic and creep components. Alternatively, they treat all the inelastic strain (including plasticity, creep, and relaxation) as a single time-dependent entity. This "unified" representation of time-dependent, inelastic strain enables these models to automatically include the observed interactions at high temperatures among plasticity, creep, and relaxation. These models, therefore, provide a more realistic and accurate description and response of the time-dependent, inelastic behavior of materials. The input of inelastic strain, calculated by using these viscoplastic models, into life prediction methods thus leads to a more accurate determination of the service life of structural components.

The mathematical framework of most viscoplastic models consists of a flow law that relates the inelastic strain rate to the deviatoric stress and evolution equations for internal state variables that represent the resistance of the material to further inelastic flow. Most models use two internal state variables, a tensor and a scalar. The tensorial and scalar variables represent the kinematic and isotropic hardenings of the material, respectively. The form of the evolution laws for the internal state variables is based on the wellknown Bailey-Orowan theory (ref. 1), which states that the high-temperature deformation of a material takes place under the influence of two competing mechanisms. One mechanism represents the hardening process with accumulated deformation, and the other represents a thermal recovery or softening process proceeding

\footnotetext{
" Resident Research Associate at Lewis Research Center.
} 
with time at temperature. The evolution rate of internal state variables is then defined as the difference between the rates of the hardening and recovery processes. Under steady-state conditions these two mechanisms balance each other.

There is a significant incentive to make viscoplastic models more realistic by incorporating into them as much material science as possible. Consequently, the mathematical structure of viscoplastic models is very complex. The constitutive equations governing the flow behavior and the evolution of internal state variables are, in general, highly nonlinear and mathematically "stiff." This inherent stiff character of the constitutive equations causes major problems during their numerical integration. Present-day structural engineering problems, such as those encountered in the aerospace and nuclear industries, involve complex thermal/ mechanical loading histories. For the solution of these problems the entire loading history has to be traced, requiring these equations to be integrated many times. The cost and computer time involved prohibit using the conventional method of employing small time steps for accurate integration of these stiff equations. There is, therefore, a strong need to develop efficient and accurate integration algorithms with a self-adaptive, time-step strategy that can achieve the desired accuracy and stability over the entire integration range (loading histories).

Keeping the aforementioned requirements in mind, numerous researchers have proposed a number of integration algorithms with self-adaptive, time-step control. These algorithms are based on explicit or implicit integration methods. Kumar et al. (ref. 2) and Banthia and Mukherjee (ref. 3) present several such integration algorithms and show that for the viscoplastic model due to Hart (ref. 4) using a simple, one-step, explicit Euler integration scheme with automatic time-step control is advantageous. Gear (ref. 5), Cormeau (ref. 6), Hughes and Taylor (ref. 7), and Hornberger and Stamm (ref. 8) have suggested special implicit integration schemes. Numerous other attempts to integrate these stiff equations have also been made, and useful information in this regard is available in the literature (refs. 9 and 10). Walker (refs. 11 and 12) and Freed et al. (ref. 13) have proposed some explicit and implicit asymptotic exponential integration algorithms and applied them to the integration of viscoplastic models. (Note that the references mentioned in this paragraph are by no means exhaustive. They are rather indicative of the importance assigned by various researchers to the development of efficient and accurate time integration strategies for use with viscoplastic models.)

We have been involved with the development of suitable integration strategies for time integration of viscoplastic models. A previous work by Arya et al. (ref. 14) reports that a simple, self-adaptive time integration strategy involving the explicit forward Euler method, although only conditionally stable, works very successfully if the time step is properly controlled at each integration step. The main advantage of using an explicit integration scheme is the ease with which it can be implemented. Another favorable aspect of using explicit integration schemes is that these do not require the assembly and inversion of Jacobian matrices. This fact is of great advantage and significance especially when the number of equations involved is large. The advantage of using implicit integration schemes is that they may allow large time steps without affecting the stability of the integration method. Cormeau (ref. 6) has, however, shown that several implicit methods suffer from the same time-step restrictions as does an explicit method, and in such circumstances the implicit integration methods offer no special advantages over the simpler explicit methods.

In light of the aforementioned facts, several explicit time integration strategies are presented in this paper. Detailed investigations were made to judge their efficiency, accuracy, and suitability for application to viscoplastic models. The integration strategies were developed and investigated in conjunction with the following four explicit integration methods:

(1) Runge-Kutta, second-order method (RK2 method)

(2) Lower Runge-Kutta method of order one (or Euler-Cauchy Method) (RK1L method)

(3) Lower Runge-Kutta method of order two (RK2L method)

(4) Exponential integration method (in conjunction with Runge-Kutta second-order method) (RK2EXP method) 
Method 1 is currently being used by the author for numerically integrating stiff equations of viscoplastic models. Methods 2 to 4 have shown promise for integrating stiff equations (refs. 15 and 16). However, their adaptability and applicability for efficiently and accurately integrating the constitutive equations of viscoplastic models is yet to be explored. The present study endeavored to do this by developing suitable time integration strategies that employ these methods.

The integration strategies presented herein are general in nature and can be applied to any viscoplastic model. However, two viscoplastic models are used to illustrate the applicability of these integration strategies. One viscoplastic model is put forth by Freed and Verrilli (ref. 17) and the other by Bodner and Partom (ref. 18). Although these two viscoplastic models possess identical mathematical frameworks, their constitutive equations have several significant differences in their forms and in the functions they involve. These differences, detailed later in the paper, assign different degrees of mathematical complexity to the models.

First, the mathematical frameworks of the viscoplastic models put forth by Freed-Verrilli and BodnerPartom are briefly described. The integration strategies developed and employed in this paper are then presented in conjunction with the four explicit integration methods listed earlier. Next, procedures to make the integration strategies self-adaptive are presented. The integration strategies are then applied to both viscoplastic models for three different types of loadings-tensile, relaxation, and cyclic. Finally, numerical results are presented and discussed in order to judge the applicability, efficiency, and accuracy of these integration strategies in conjunction with the viscoplastic models.

\section{VISCOPLASTIC MODELS}

\section{Freed-Verrilli Viscoplastic Model}

Following the general mathematical structure adopted by most viscoplastic models and as mentioned earlier, the Freed-Verrilli (F-V) viscoplastic model (ref. 17) also incorporates two internal state variables. One a tensorial variable called the backstress, accounts for strain-induced or kinematic hardening. The other, called the drag stress (strength), represents isotropic hardening and is a scalar variable. The model is based on the assumption that there is no explicit coupling between the dynamic and static recovery terms in an evolutionary equation for the internal state. This assumption renders a simpler mathematical framework for the model. The model also employs a small displacement and a small strain formulation.

The total strain rate $\dot{\varepsilon}_{i j}$ is assumed to be composed of elastic $\dot{\varepsilon}_{i j}^{e}$, inelastic (including plasticity, creep, and relaxation) $\dot{\varepsilon}_{i j}^{v}$, and thermal $\dot{\varepsilon}_{i j}^{t}$, strain rate components and can be written as

$$
\dot{\varepsilon}_{i j}=\dot{\varepsilon}_{i j}^{e}+\dot{\varepsilon}_{i j}^{v}+\dot{\varepsilon}_{i j}^{l}, \quad \text { where } i, j=1,2,3
$$

In equation (1), the symbol $\varepsilon$ denotes the strain and the dot over the symbol indicates its derivative with respect to time $t$. Superscripts $e, v$, and $t$ refer to elastic, viscoplastic (inelastic), and thermal components of the strain rate.

For an isotropic material the elastic strain rate $\dot{\varepsilon}_{i j}^{e}$ is related to the stress rate $\dot{\sigma}_{i j}$ by Hooke's law

$$
\dot{\varepsilon}_{i j}^{e}=\frac{1+v}{E} \dot{\sigma}_{i j}-\frac{v}{E} \dot{\sigma}_{k k} \delta_{i j}
$$


where $E$ is Young's modulus, $v$ is Poisson's ratio, and $\delta_{i j}$ is the Kronecker delta. The repeated subscripts in equation (2) and elsewhere imply summation over their range.

The deviatoric stress $S_{i j}$ is defined by

$$
S_{i j}=\sigma_{i j}-\frac{1}{3} \sigma_{k k} \delta_{i j}
$$

The effective stress $\Sigma_{i j}$ has the expression

$$
\Sigma_{i j}=S_{i j}-B_{i j}
$$

where $B_{i j}$ is the backstress.

Flow law.-Defining the invariant $J_{2}$ by

$$
J_{2}=\frac{1}{2} \Sigma_{i j} \Sigma_{i j}
$$

the flow law is written as

$$
\dot{\varepsilon}_{i j}^{v}=\theta Z \frac{\Sigma_{i j}}{J_{2}}
$$

The function $\theta$, called the thermal diffusivity function, contains the temperature dependence of the model. It is defined by

$$
\theta= \begin{cases}\exp \left(-\frac{Q}{k T}\right), & \text { if } T \geq 0.5 T_{m} \\ \exp \left[-\frac{2 Q}{k T_{m}}\left\{\ln \left(\frac{T_{m}}{2 T}\right)+1\right\}\right], & \text { if } T \leq 0.5 T_{m}\end{cases}
$$

$Q$ is the activation energy, $k$ is the Boltzmann constant, $T$ is the absolute temperature, and $T_{m}$ is the melting point of the material.

The function $Z$, called the Zener-Hollomon parameter, is given as

$$
Z= \begin{cases}A F^{n}, \quad \text { if } F \leq 1 \\ A \exp [n(F-1)], & \text { if } F \geq 1\end{cases}
$$

and $A$ and $n$ are material constants. The function $F$ is defined as 


$$
F=\frac{J_{2}}{D}
$$

where $D$ denotes the drag stress.

Evolution laws.-The differential equations that govern the growth of the internal state variables (backstress $B_{i j}$ and drag stress $D$ ) are

$$
\dot{B}_{i j}=H\left(\dot{\varepsilon}_{i j}^{v}-\frac{B_{i j}}{L} I_{2}\right)
$$

and

$$
\dot{D}=h\left[\frac{I_{2}}{G}-\theta r(G)\right]
$$

where

$$
I_{2}=\theta Z
$$

In equations (10) and (11), $H, h$, and $L$ are inelastic material constants. The constant $L$ denotes the limiting value of the backstress at kinematic saturation (ref. 17). The recovery function $r$ is defined by using the following equations:

$$
\begin{gathered}
r(G)= \begin{cases}0, & \text { if } D=D_{0} \\
R(G), & \text { if } D>D_{0}\end{cases} \\
R(G)= \begin{cases}A G^{n-1}, & \text { if } G \leq 1 \\
A \exp [n(G-1)] / G, & \text { if } G \geq 1\end{cases}
\end{gathered}
$$

and

$$
G=\frac{L}{S-D}
$$

where $S$ and $D_{0}$ are material constants.

For the theory to be thermodynamically admissible, the dissipativity condition must be satisfied. This results in the following constraint on the function $r$ : 


$$
r \geq Z\left[\frac{1}{G}-2\left(F+\frac{B_{2}^{2}}{L D}\right)\right]
$$

where

$$
B_{2}=\frac{1}{2} B_{i j} B_{i j}
$$

The values of elastic and inelastic material constants appearing in the preceding equations are given in table I for copper (ref. 17).

\section{Bodner-Partom Viscoplastic Model}

The viscoplastic model put forth by Bodner (ref. 19) in 1968 is one of the first unified constitutive models that does not require an explicit yield function (or loading/unloading conditions) to govern the inelastic response of a material. The mechanistic basis for the model development stems from the work of Gilman (ref. 20) and Johnston and Gilman (ref. 21). In the Bodner-Partom (B-P) model the inelastic strain rate is considered to be a function of the current value of stress $\sigma$ and an internal state variable that represents a measure of hardening or resistance to inelastic flow. Although the general mathematical framework of this viscoplastic model is akin to that adopted by other viscoplastic models, it has several significant differences from them. For example, in this model the inelastic strain rate is expressed as a function of stress $\sigma$ and not as a function of overstress, which is defined as the difference of stress $\sigma$ and the backstress. Also, the measure of inelastic hardening in this model is taken to be inelastic (plastic) work and not the inelastic strain as is done in other viscoplastic models.

Several modifications to this model have been proposed from time to time. The modifications of the model advanced by Bodner and Partom (refs. 18 and 22) included large deformations and isotropic hardening, respectively. Stouffer and Bodner (refs. 23 and 24) included the Bauschinger effect associated with load reversals and the generally anisotropic nature of inelastic hardening. Bodner (ref. 25) extended the model to include evolution of damage. The model used in the present paper (from ref. 18) includes isotropic and directional hardening, thermal recovery of hardening, and general temperature dependence of inelastic flow. The constitutive equations of the model are given here.

Flow law.-The total strain rate is again assumed to be the sum of elastic, inelastic (viscoplastic), and thermal strain rate components, and the inelastic strain rate is assumed to have the form of the PrandtlReuss flow law. In symbols

$$
\begin{gathered}
\dot{\varepsilon}_{i j}=\dot{\varepsilon}_{i j}^{e}+\dot{\varepsilon}_{i j}^{v}+\dot{\varepsilon}_{i j}^{l}, \quad \text { where } i, j=1,2,3 \\
\dot{\varepsilon}_{i j}^{v}=\lambda S_{i j} ; \quad \dot{\varepsilon}_{k k}^{v}=0
\end{gathered}
$$

where

$$
S_{i j}=\sigma_{i j}-\frac{1}{3} \delta_{i j} \sigma_{k k}
$$

In these equations the symbols $\varepsilon, S, \sigma$, and $\delta$ and the superscripts $e, v$, and $t$ have the same meaning as defined earlier. A repeated index implies summation over its range, and the parameter $\lambda$ is defined later. 
Kinetic equation.-The inelastic deformations are governed by the kinetic relation

$$
D_{2}^{p}=D_{0}^{2} \exp \left\{-\left[Z^{2} /\left(3 J_{2}\right)\right]^{n}\right\}
$$

with

$$
Z=Z^{I}+Z^{D} ; \quad D_{2}^{p}=\frac{1}{2} \varepsilon_{i j}^{v} \varepsilon_{i j}^{v}
$$

and

$$
J_{2}=\frac{1}{2} S_{i j} S_{i j} ; \quad \lambda^{2}=D_{2}^{p} / J_{2}
$$

In these equations $Z$ is an internal state variable that represents the resistance of the material to inelastic flow. This state variable in the model is assumed to be composed of isotropic hardening $Z^{I}$ and directional hardening $Z^{D}$ components. Also, $D_{0}$ denotes the limiting strain rate in shear and $n$ is a material constant.

Isotropic-hardening evolution laws. - The growth or evolution of the isotropic-hardening component is governed by

$$
\dot{Z}^{I}=m_{1}\left(Z_{1}+\alpha Z_{3}-Z^{I}\right) \dot{W}_{p}-A_{1} Z_{1}\left[\left(Z^{I}-Z_{2}\right) / Z_{1}\right]^{r_{1}}
$$

where

$$
\begin{gathered}
\dot{\alpha}=m_{2}\left(\alpha_{1}-\alpha\right) \dot{W}_{p} \sin \theta \\
\theta=\cos ^{-1}\left(V_{i j} \bar{V}_{i j}\right) \quad \text { or } \quad \theta=\cos ^{-1}\left(u_{i j} \bar{u}_{i j}\right) \\
V_{i j}=\beta_{i j} / \sqrt{\beta_{k l} \beta_{k l}} ; \quad \bar{V}_{i j}=\dot{\beta}_{i j} / \sqrt{\dot{\beta}_{k l} \dot{\beta}_{k l}} \\
u_{i j}=\sigma_{i j} / \sqrt{\sigma_{k l} \sigma_{k l}} ; \quad \bar{u}_{i j}=\dot{\sigma}_{i j} / \sqrt{\dot{\sigma}_{k l} \dot{\sigma}_{k l}}
\end{gathered}
$$

with

$$
Z^{I}(0)=Z_{0} ; \dot{W}_{p}=\sigma_{i j} \dot{\varepsilon}_{i j}^{\nu} ; W_{p}(0)=0 ; \alpha(0)=0
$$

In these equations $Z_{0}$ is the initial value of the isotropic hardening variable $Z^{l} ; Z_{1}$ denotes the limiting (maximum) value of $Z^{I} ; Z_{2}$ is the fully recovered (minimum) value of $Z^{I}$; and $Z_{3}$ denotes the limiting (maximum) value of $Z^{D}$. The parameter $\theta$ is a measure of nonproportionality of loading, and $\alpha$ is a loadinghistory-dependent variable that provides an additional hardening increment in the evolution equation for $Z I$. 
The coefficient $\alpha_{1}$ corresponds to the limiting value of $\alpha$, and $m_{1}$ and $m_{2}$ denote the hardening rate coefficients of $Z^{l}$ and $Z^{D}$, respectively. The rate of inelastic (plastic) work is denoted by $\dot{W}_{p}$. The symbols $A_{1}$ and $r_{1}$ denote the recovery coefficient and the recovery exponent for $Z l$. The second-order symmetric tensor $\beta_{i j}$ represents the directional-hardening state variable.

Directional-hardening evolution law.-The evolution equation for the directional-hardening variable $\beta_{i j}$ has the same general form as that for isotropic hardening. It, however, has a tensorial character. It is written as

$$
\dot{\beta}_{i j}=m_{2}\left(z_{3} u_{i j}-\beta_{i j}\right) \dot{W}_{p}-A_{2} Z_{1}\left(\sqrt{\beta_{k l} \beta_{k l}} / Z_{1}\right)^{2} V_{i j}
$$

with

$$
Z^{D}=\beta_{i j} u_{i j} ; Z^{D}(0)=0 ; \beta_{i j}(0)=0
$$

Here $m_{2}$ denotes the hardening rate coefficient of $Z^{D}$. The symbols $A_{2}$ and $r_{2}$ represent the recovery coefficient and the recovery exponent for $Z^{D}$. In addition to elastic material constants the model has the following inelastic material constants: $D_{0}, Z_{0}, Z_{1}, Z_{2}, Z_{3}, m_{1}, m_{2}, \alpha_{1}, A_{2}, r_{1}, r_{2}$. and $n$. In most cases one can set $r_{1}=$ $r_{2}, A_{1}=A_{2}$, and $Z_{0}=Z_{2}$. The values of all these constants for the material B $1900+H f$, taken from reference 18 , are listed in table II.

\section{NUMERICAL INTEGRATION METHODS}

To develop efficient, accurate, and stable explicit integration strategies for integrating the constitutive (differential) equations of the F-V and B-P viscoplastic models, four explicit numerical integration methods were considered. The reasons for considering only the explicit integration methods have been explained in the introduction. To keep the presentation simple, the mathematical forms of the integration strategies have been presented for only one differential equation. Generalization of these integration strategies for a system of differential equations is straightforward and presented subsequently.

Consider the first-order differential equation

$$
\frac{d y}{d t}=f(t, y)
$$

Let the solution, denoted by $y$, of this differential equation be known at time $t$. The mathematical formulas for the four methods are given here to calculate the solution at time $t+h$ where $h$ denotes the increment in time $t$.

\section{Runge-Kutta, Second-Order Method (or RK2 Method)}

For equation (32) the approximate solution at $t+h$ using the Runge-Kutta method is given by the wellknown expressions (see, e.g., ref. 26)

$$
y(t+h)=y+\frac{1}{2}\left(k_{1}+k_{2}\right)
$$


with

$$
k_{1}=h f(t, y) ; \quad k_{2}=h f\left(t+h, y+k_{1}\right)
$$

\section{Lower Order Runge-Kutta Methods (RK2L and RK1L Methods)}

Fehlberg (ref. 15) has proposed several lower order Runge-Kutta formulas for the solution of a system of ordinary differential equations and has applied them to heat transfer problems. He has shown for these problems that the low-order, Runge-Kutta methods are considerably faster and of equal or better accuracy than the conventional explicit integration formulas. Higher order Runge-Kutta formulas offer no special advantages because the increase in the integration step size is restricted by the stability considerations resulting from the exponential character of the solution.

It is well established that small truncation errors lead to efficient Runge-Kutta integration formulas and that the permissible integration step size is inversely related to the magnitude of these truncation errors. The attempt in the lower order integration formulas proposed by Fehlberg has, therefore, been to keep the truncation errors as small as possible. In this study we selected two lower order Runge-Kutta methods proposed by Fehlberg: the lower Runge-Kutta method of order two (or RK2L method) and the lower Runge-Kutta method of order one (the Euler-Cauchy or RK1L method)). The general formula for a lower Runge-Kutta method of order $n$ is given next. be written as

Following Fehlberg (ref. 15), the integration formulas for a lower Runge-Kutta method of order $n$ can

$$
y(t+h)=y(t)+h \sum_{\kappa=0}^{n} c_{\kappa} f_{\kappa}
$$

and

$$
f_{\mathrm{\kappa}}=f\left(t+\alpha_{\kappa} h, y+\sum_{\lambda=0}^{\mathrm{k}-1} \beta_{\mathrm{\kappa} \lambda} f_{\lambda}\right)
$$

The coefficients $\alpha_{k}, \beta_{k \lambda}$, and $c_{k}$ are determined by using Taylor's expansion. The values of these coefficients for lower order Runge-Kutta methods of orders one and two, taken from Fehlberg (ref. 15), are listed in tables III and IV. Complete derivation of these formulas and estimation of the coefficients may be found in this reference.

\section{Exponential Integration Method (or RK2EXP Method)}

Several explicit and implicit exponential integration algorithms have been proposed from time to time for the integration of the "stiff" constitutive equations of viscoplastic models (refs. 11 to 13). These exponential integration algorithms are claimed to be considerably more stable (explicit algorithms) or unconditionally stable (implicit algorithms) in the "stiff" region of integration provided that the integrand satisfies certain mathematical requirements. Applying some implicit exponential integration algorithms to stress analysis of structural engineering problems involving viscoplastic models (ref. 27) has shown them to be more efficient than the conventional integration methods.

Explicit integration algorithms offer several advantages for the stress analysis of structural engineering problems involving many degrees of freedom. Therefore, an integration strategy based on an explicit 
exponential integration method (ref. 16) was developed for application to structural (and life) analysis problems involving viscoplastic models. The exponential integration method developed in reference 16 for use in conjunction with the explicit Runge-Kutta method is shown to possess better stability characteristics than the conventional explicit integration methods. A built-in capability of the method automatically determines whether the problem is stiff or nonstiff and efficiently integrates it accordingly. This exponential integration algorithm has successfully been applied to several mathematically stiff problems and shown to be very efficient. A brief description and derivation of this integration method is followed by its numerical implementation and the description of the integration strategy for application to viscoplastic models.

Rigorous mathematical details are avoided to keep the presentation simple. Readers interested in complete details may find these in reference 16 .

Integrating equation (32) from $t$ to $t+h$ yields

$$
y(t+h)=y(t)+\int_{s=t}^{t+h} f[s, y(s)] d s
$$

This is the fundamental theorem of integral calculus.

To develop an exponential integration scheme, start with the Laplace integral

$$
I=\int_{0}^{A} e^{-x s} F(s) d s, \quad \text { where } x>0
$$

and $F(s)$ is of the exponential order. Write

$$
F(s)=e^{-m s} F(s) e^{m s}
$$

or

$$
F(s)=e^{-m s}\left\{F(0)+\left[F^{\prime}(0)+m F(0)\right] s+\mathrm{O}\left(s^{2}\right)\right\}
$$

by using Mclaurin' 12 s expansion. Choose $m$ such that

$$
F^{\prime}(0)+m F(0)=0 \text { or } m=-F^{\prime}(0) / F(0)
$$

Therefore,

$$
I=\int_{0}^{A} e^{-(x+m) s} F(s) e^{m s} d s
$$

Integrating gives

$$
I \approx \frac{F(0)}{x+m}\left[1-e^{-(x+m) A}\right]
$$

This result is used to accomplish the exponential integration of equation (32). 
Substituting $s=t+u$ into equation (35) yields

$$
y(t+h)=y(t)+\int_{u=0}^{h} e^{-m u} y^{\prime}(t+u) e^{m u} d u
$$

Also

$$
e^{m u} y^{\prime}(t+u)=y^{\prime}(t)+\left[y^{\prime \prime}(t)+m y^{\prime}(t)\right] u+\mathrm{O}\left(u^{2}\right)
$$

Again, choose $m$ such that

$$
y^{\prime \prime}(t)+m y^{\prime}(t)=0 \text { or } m=-y^{\prime \prime}(t) / y^{\prime}(t)
$$

Using equations (42) to (45) gives

$$
y(t+h)=y(t)+y^{\prime}(t) \frac{1-e^{-m h}}{m}
$$

For $m>0$, equation (46) yields an approximation to equation (32) that is useful for a large $m$ or a small $h$. For $m \leq 0$ and decaying exponential (stiff) behavior this method is not applicable. In this case use an explicit integration (e.g., second-order Runge-Kutta) method as the nonstiff integration method. It is important to note that by examining the sign of the exponent $m$ the RK2EXP method automatically determines whether the given differential equation is stiff $(m>0)$ or nonstiff $(m \leq 0)$ in the region of integration and integrates it accordingly.

\section{NUMERICAL IMPLEMENTATION}

The procedure for implementing the exponential integration method is given here in a step-by-step form:

(1) Calculate derivatives at the start of the step.

$$
f[t, y(t)] \equiv d_{\text {old }}
$$

(2) Calculate the Euler values.

$$
z(t+h)=y(t)+h f[t, y(t)]
$$

(3) Calculate the new derivatives.

$$
f[t+h, z(t+h)] \equiv d_{\text {new }}
$$

Note that these calculations are required also for the classical Runge-Kutta method.

$$
\text { If } d_{\text {old }} \neq 0 \text {, }
$$

Calculate $m=\left(d_{\text {old }}-d_{\text {new }}\right) /\left(h \times d_{\text {old }}\right)$ 


$$
\begin{aligned}
& \text { If } m>0 \text {, } \\
& \text { Calculate the exponential approximation } \\
& y(t+h)=y(t)+d_{\text {old }} \times(1-\exp (-m \times h) / m \\
& \text { Endif } \\
& \text { Endif } \\
& \text { If } d_{\text {old }}=0 \text { or } m \leq 0 \text {, } \\
& \text { Calculate the RK2 approximation } \\
& y(t+h)=y(t)+(h / 2) \times\left(d_{\text {old }}+d_{\text {new }}\right)
\end{aligned}
$$

Endif

\section{ESTIMATION OF LOCAL ERROR (IMBEDDING TECHNIQUE)}

To estimate the local error in the solution, an "imbedding" technique is employed. In this technique the local error is defined as the difference in the solutions obtained by using two methods of different orders. For the RK2 method the local error is the difference between the RK2 and readily available Euler values. For the exponential method it is estimated by employing the exponential approximation (see ref. 16)

$$
y_{a}(t+h)=y(t) \exp \left[y^{\prime}(t) h / y(t)\right]+\mathrm{O}\left(h^{2}\right)
$$

where the subscript $a$ refers to an approximation to the integration method.

\section{AUTOMATIC TIME-STEP INTEGRATION}

Two schemes were adopted to make the integration strategy self-adaptive, that is, capable of automatically picking suitable time steps that preserve the stability and accuracy of the solution:

(1) Scheme I. Employed herein, scheme I defines the error in the solution during the current time step as

$$
E_{R}=\frac{|\Delta y|}{|y|}
$$

(2) Scheme II. It defines the error in the solution during the current time step as

$$
E_{R}=\frac{\left|y-y_{a}\right|}{|y|}
$$

Generalization to a system of $n$ equations is easily obtained by defining an error norm $e$ as

$$
e=\left(\frac{\sum_{i=1}^{n} E_{R_{i}} E_{R_{i}}}{n}\right)^{1 / 2}
$$


The integration strategy runs as follows:

(1) Prescribe the upper $e_{u}$ and lower $e_{l}$ limits for the error norm $e$.

(2) If $e>e_{u}$, halve the time step to $h / 2$. (If necessary, repeat this until a value of $h$ is obtained that satisfies the condition $e \leq e_{u}$.)

(3) If $e<e_{l}$, double the current time step to $2 h$.

(4) If $e_{l} \leq e \leq e_{u}$, retain the current time step.

Proceed to the next step calculations with the time step rendered by the preceding and so on.

\section{APPLICATIONS}

To illustrate the applications of the self-adaptive time integration strategy in conjunction with the four explicit integration methods mentioned earlier, computer programs in FORTRAN were developed and applied to several uniaxial problems. The uniaxial problems considered consisted of tensile, relaxation, and cyclic thermal/mechanical loadings.

Figures 1(a), (b), and (c) exhibit tensile, relaxation, and cyclic loadings for the F-V model. The tensile loading (fig. 1(a)) consisted of a stress rate of $6.10 \times 10^{-3} \mathrm{MPa} / \mathrm{s}$ at $149^{\circ} \mathrm{C}$. For relaxation loading (fig. 1(b)) the specimen was deformed to a strain of 0.015 in $1000 \mathrm{~s}$ and then allowed to relax at the same strain for $10000 \mathrm{~s}$. For in-phase cyclic thermomechanical loading (fig. l(c)) the specimen was cycled between \pm 0.00375 strain in $1000 \mathrm{~s}$. The temperature of the specimen was cycled (in phase with the strain) between $200^{\circ} \mathrm{C}$ (at -0.00375 strain) and $500{ }^{\circ} \mathrm{C}$ (at 0.00375 strain) in $1000 \mathrm{~s}$.

The tensile, relaxation, and cyclic thermal/mechanical loadings for the B-P model are shown in figures 2(a), (b), and (c), respectively. The tensile loading (fig. 2(a)) consisted of loading the specimen to $0.010 \mathrm{strain}$ in $120 \mathrm{~s}$ at $871^{\circ} \mathrm{C}$. For relaxation loading (fig. 2(b)) the specimen was loaded to 0.008 strain in approximately $100 \mathrm{~s}$ at $871^{\circ} \mathrm{C}$ and then allowed to relax for $1000 \mathrm{~s}$. For cyclic thermal/ mechanical loadings (fig. 2(c)) the strain and temperature were cycled in phase between \pm 0.004 and 538 and $760^{\circ} \mathrm{C}$, respectively. The time for one cycle was $160 \mathrm{~s}$.

\section{RESULTS AND DISCUSSION}

The constitutive equations of the F-V and B-P viscoplastic models were integrated in conjunction with the Runge-Kutta second-order method (RK2), the lower Runge-Kutta method of order one or the EulerCauchy (RK1L), the lower Runge-Kutta method of order two (RK2L), and the exponential (RK2EXP) integration method. The respective tensile, relaxation, and cyclic thermal/mechanical loadings were considered in numerical computations for the F-V and B-P models. The results of these computations are shown in figure 3 for the F-V model and in figure 4 for the B-P model.

These computations were performed to ensure that the implementation of the self-adaptive integration strategy in conjunction with these four explicit integration methods yielded accurate results. The computations presented in figures 3 and 4 were carried out by assigning very small error tolerances for integration for different types of loadings (described earlier). The curves marked as "exact" in these figures were obtained by using the Runge-Kutta, second-order method (without self-adaptive integration strategy) and choosing a very small time step for integration of the constitutive differential equations of the two viscoplastic models. Figures 3 and 4 show that for both viscoplastic models and all uniaxial problems (tensile, relaxation, and cyclic loadings), the self-adaptive integration strategies produced results that are in close agreement with the "exact" results. This conclusion gives confidence in accurate implementation of the self-adaptive integration strategies for the four explicit integration methods.

To test the efficiency of different numerical integration algorithms in conjunction with the selfadaptive time integration strategy described earlier, a large number of cases, involving the same loadings and viscoplastic models, were run. Different values and combinations of error tolerances were considered in numerical computations involving different types of loadings. Because it is not possible to list all the 
results, the most significant results from these calculations for the three types of loadings are given in table $\mathrm{V}$ for the F-V model and in table VI for the B-P model.

A criterion must be established for comparing the efficiencies and accuracies of different integration algorithms. The following criterion was adopted in the present study. First, an arbitrary and desired accuracy (or acceptable error) in the solution was assumed. Then, the error tolerances for each integration algorithm and error control scheme were varied until the required accuracy was achieved in the solution. This was done for each type of loading and viscoplastic model. The execution time (CPU time) for each case was recorded. The execution time for tracing a given loading path when using the RK2EXP (or any other) integration algorithm depended not only on the error tolerances but also on the scheme being used to define the time steps during the integration. Of the execution times thus obtained, the smallest time for each integration algorithm and the corresponding error tolerances, scheme, etc., were selected. These times are listed in tables V and VI for all loadings and viscoplastic models. These tables readily reveal the most efficient integration algorithm for a given viscoplastic model and given loading.

It is advantageous to define the format of tables $\mathrm{V}$ and VI at this stage. The tables have identical formats. Column 1 lists the integration algorithms investigated. Column 2 indicates which error norm (scheme), equation (48) or equation (49), was used to control the time step for the self-adaptive time integration strategy. The prescribed upper error limits $\mathrm{RTOL}_{u}$ for the Runge-Kutta methods and $\mathrm{ETOL}_{u}$ for the exponential integration method are shown in columns 3 and 4 , respectively. These values represent the value of $e_{u}$ (see section Automatic Time-Step Integration) for the two integration methods. An additional advantage of using the RK2EXP method, as may be noted from columns 3 and 4, is that it allows various combinations of error tolerances $\mathrm{RTOL}_{u}$ and $\mathrm{ETOL}_{u}$, making the RK2EXP integration method more versatile than the other integration methods. Column 5 shows the total number of steps (iterations) required by a given method to trace the given loading path (cycle). The number of steps for the RK2EXP method in column 5 is split into two parts, $a$ and $b$. The superscript a on a number denotes the number of times the RK2 method was used, and the superscript $b$ denotes the number of times the exponential method was used. Column 6 lists the execution times (CPU times) in seconds (on a Cray-YMP computer) taken by various integration methods to trace a given loading path. Finally, the accuracy of a given integration method is indicated in column 7 . This column lists the error in the result obtained by using a given integration algorithm. The error is defined as

$$
\text { Error }=\left(Y_{c}-Y_{e}\right) / Y_{e}
$$

where $Y_{c}$ denotes the solution obtained by using the current integration algorithm and $Y_{e}$ designates the "exact" solution obtained by using the RK2 method with a constant and small time step as described earlier.

\section{Results Using Freed-Verrilli Model}

Tensile loading.-The tensile loading shown in figure 1 (a) and the F-V model were used to generate the results listed in table V(a). The RK1L and RK2L integration algorithms were found to be less efficient than the RK2 method. The execution times for the former two methods were 10.002 and $12.949 \mathrm{~s}$, respectively, whereas for the same accuracy $\left(0.460 \times 10^{-3}\right)$ the time taken by the RK2 method was $9.871 \mathrm{~s}$. The most interesting result for the tensile loading case is obtained from the fourth row of the table. It shows the RK2EXP method to be significantly more efficient (by a factor of about 2) and slightly more accurate $\left(0.416 \times 10^{-3}\right)$ than the RK2 method $\left(0.460 \times 10^{-3}\right)$. Fewer iterations $(40352)$ were performed by the RK2EXP method to trace the tensile loading path than by RK2 and the other integration methods (104 091).

Relaxation loading.-The results of integrating the F-V model over the relaxation loading path of figure 1(b) by the different integration methods are shown in table V(b). For the same accuracy the RK1L and RK2L methods were comparable to or less efficient than the RK2 method. The RK2EXP method, for a comparable accuracy, took less execution time (1.096 s) than the RK2 method (1.205 s). The RK2EXP method was about 10 percent more efficient (faster) than the RK2 method and took fewer steps (8381) 
than the RK2 method (10 802). Using the RK2EXP method in this case is clearly advantageous as, for comparable accuracy, it is more efficient than the RK2 method.

Cyclic loading.-Table V(c) summarizes results for the cycling loading (see fig. 1(c)) obtained with the F-V model. The RK2, RK1L, and RK2L integration methods gave almost the same accuracy (column 7). However, RK1L and RK2L were not as efficient as the RK2 method. The execution times of 3.554 and $4.487 \mathrm{~s}$ for the RK1L and RK2L methods were higher than that for the RK2 method ( $3.508 \mathrm{~s}$ ). Comparing the results for the RK2 and RK2EXP methods (rows 1 and 5) shows that the RK2EXP method took less execution time ( $3.259 \mathrm{~s})$ than the RK2 method (3.508 s). However, the error was larger $\left(0.343 \times 10^{-3}\right)$ for RK2EXP than for RK2 $\left(0.227 \times 10^{-3}\right)$. Therefore, for cyclic loading the RK2 and RK2EXP methods are comparably advantageous and either may be used.

\section{Results Using Bodner-Partom Model}

Tensile loading.-The results for the tensile loading of figure 2(a) and the B-P model are shown in table VI(a). The total numbers of iterations performed using the RK2, RK1L, and RK2L integration methods were the same. However, the RK2EXP integration method took only 2129 steps to trace the given tensile loading path. The most important results of the tensile loading case are obtained from columns 6 and 7. Column 6 shows that for the present case the RK2EXP method took significantly less execution time $(0.223 \mathrm{~s})$ than the RK2 (1.166 s), RK1L (1.183 s), and RK2L (1.620 s) integration methods. Also the RK2EXP method yielded a more accurate solution (error of $0.104 \times 10^{-3}$ ) than the RK2 method (error of $0.174 \times 10^{-3}$ ). Therefore, using the RK2EXP method to integrate the constitutive equations of the B-P model is of significant advantage in this case.

Relaxation loading.-Table VI(b) displays the results for relaxation loading (see fig. 2(b)) obtained by the different integration methods. For a given accuracy the execution times were higher for the RK1L and RK2L integration methods than for the RK2 method. The execution time was also marginally higher for the RK2EXP method $(6.653 \mathrm{~s})$ than for the RK2 method $(6.270 \mathrm{~s})$. The total numbers of iterations performed by the different integration methods to trace the same relaxation loading path were comparable. In this case the RK2EXP method was found (at best) to be comparable to the RK2 method.

Cyclic loading.-The results of integrating the constitutive equations of the B-P model for different integration methods are listed in table VI(c) for the cyclic loading of figure 2(c). For a given accuracy the execution times were higher for the RK1L, RK2L, and RK2EXP integration methods than for the RK2 integration method. The total number of iterations performed for the RK2, RK1L, and RK2L methods were the same (96 058). However, for the RK2EXP method, it was 96059 . The RK2EXP method does not offer any particular advantage with regard to efficiency and accuracy in this case.

\section{CONCLUSIONS}

The results presented for both viscoplastic models and three types of loadings show that, for comparable accuracy, the lower Runge-Kutta methods of orders one and two are less efficient than the Runge-Kutta, second-order and exponential integration methods. The lower Runge-Kutta methods are, therefore, not advantageous for application to viscoplastic models. The results also show that, for comparable accuracy, the efficiency of the integration algorithms depends on the type of loading. For example, for tensile loading, for comparable accuracy and for both viscoplastic models, the exponential integration method is much faster than the Runge-Kutta, second-order method. However, for the relaxation and cyclic loadings and for comparable accuracy, the exponential method is at best as efficient as the Runge-Kutta, second-order method.

Because, in general, for both viscoplastic models and all the loadings considered, the exponential integration algorithm provides significantly higher (tensile loading) or comparable (relaxation and cyclic loadings) efficiency of any other integration algorithm, its use for integrating the "stiff" equations of viscoplastic models may be advantageous. Note that the results presented in this paper pertain to uniaxial loadings and uniaxial forms of the viscoplastic models. Computer programs in FORTRAN were developed 
to test the efficiency and accuracy of various integration algorithms with self-adaptive strategy. The structural engineering problems, however, involve multiaxial loadings and forms of the viscoplastic models. To judge the efficiency and accuracy of the exponential integration algorithm for application to these problems, it should be implemented into a finite element code. The process of implementing the exponential integration algorithm with self-adaptive strategy into a finite element code is in progress. The results of this work will be reported subsequently.

\section{REFERENCES}

1. Orowan, E.: The Creep of Metals. J. W. Scotl. Iron Steel Inst., vol. 54, 1946, pp. 45-53.

2. Kumar, V.; Morjaria, M.; and Mukherjee, S.: Numerical Integration of Some Stiff Constitutive Models of Inelastic Deformation. ASME J. Eng. Matls. Technol., vol. 102, 1980, pp. 92-96.

3. Banthia, V.; and Mukherjee, S.: On an Improved Time Integration Scheme for Stiff Constitutive Models of Inelastic Deformation. ASME J. Eng. Matls. Technol., vol. 107, 1985, pp. 282-285.

4. Hart, E.W.: Constitutive Relations for the Nonelastic Deformation of Metals. ASME J. Eng. Matls. Technol., vol. 98, 1976, pp. 97-105.

5. Gear, C.W.: Algorithm 407 DIFSUB for Solution of Ordinary Differential Equations. Communications of the ACM., vol. 14, 1971, pp. 176-179.

6. Cormeau, I.: Numerical Stability in Quasi-Static Elastic/Viscoplasticity. Int. J. Numer. Methods in Eng., vol. 1, 1975, pp. 109-127.

7. Hughes, T.J.R.; and Taylor, R.L.: Unconditionally Stable Algorithms for Quasi-Static Elasto/ Viscoplastic Finite Element Analysis. Computers and Structures, vol. 8, 1978, pp. 169-173.

8. Hornberger, K.; and Stamm, H.: An Implicit Integration Algorithm With a Projection Method for Viscoplastic Constitutive Equations. Int. J. Numer. Methods in Eng., vol. 28, 1989, pp. 2397-2421.

9. Lambert, J.D.: Computational Methods in Ordinary Differential Equations. Wiley, London, 1973.

10. Roberts, C.E. (Jr.): Ordinary Differential Equations - A Computational Approach. Prentice-Hall, 1979.

11. Walker, K.P.: Research and Development Program for Nonlinear Structural Modelling With Advanced Time-Temperature Dependent Constitutive Relationships. NASA CR-165533, 1981.

12. Walker, K.P.: A Uniformly Valid Asymptotic Integration Algorithm for Unified Constitutive Models. Advances in Inelastic Analysis. S. Nakazawa et al., eds., ASME, New York; AMD-Vol. 28 \& PED-Vol. 28, 1987, pp. 13-27.

13. Freed, A.D.; Yao, M.; and Walker, K.P.: Asymptotic Integration Algorithms for First Order ODE's With Application to Viscoplasticity. NASA TM-105587, 1992.

14. Arya, V.K.; Hornberger, K.; and Stamm, H.: On the Numerical Integration of Viscoplastic Models. Report No. KfK-4082, Kernforschungszentrum, Karlsruhe, Germany, 1986.

15. Fehlberg, E.: Low-Order Classical Runge-Kutta Formulas With Stepsize Control and Their Application to Some Heat Transfer Problems. NASA TR R-315, 1964.

16. Ashour, S.S.; and Hanna, O.T.: Explicit Exponential Method for the Integration of Stiff Ordinary Differential Equations. AIAA J. Guidance, vol. 14, 1991, pp. 1234-1239.

17. Freed, A.D.; and Verrilli, M.: A Viscoplastic Theory Applied to Copper. NASA TM-100831, 1988. 
18. Bodner, S.R.; and Partom, Y.: Constitutive Equations for Elastic-Viscoplastic Strain Hardening Materials. ASME J. Appl. Mech., vol. 42, 1975, pp. 385-389.

19. Bodner, S.R.: Constitutive Equations for Dynamic Material Behavior. Mechanical Behavior of Materials Under Dynamic Loads, U.S. Lindholm, ed., Springer-Verlag, New York, 1968.

20. Gilman, J.J.: Dislocation Mobility in Crystals. J. Appl. Phys., vol. 36, 1964, p. 3195.

21. Johnston, W.G. and Gilman, J.J.: Dislocation Velocities, Dislocation Densities and Plastic Flow in Lithium Fluoride Crystal. J. Appl. Phys., vol. 30, 1959, pp. 129-144.

22. Bodner, S.R.; and Partom, Y.: A Large Deformation Elastic-Viscoplastic Analysis of a Thick-Walled Spherical Shell. ASME J. Appl. Mech., vol. 39, 1972, pp. 751-757.

23. Stouffer, D.C.; and Bodner, S.R.: A Constitutive Model for the Deformation Induced Anisotropic Plastic Flow of Materials. Int. J. Eng. Sci., vol. 17, 1979, pp. 757-764.

24. Bodner, S.R.; and Stouffer, D.C.: Comments on Anisotropic Plastic Flow and Incompressibility. Int. J. Eng. Sci., vol. 21, 1983, pp. 211-215.

25. Bodner, S.R.: Evolution Equations for Anisotropic Hardening and Damage of Elastic-Viscoplastic Materials. Plasticity Today-Modelling, Methods and Applications, A. Sawczuk and G. Bianchi, eds., Elsevier Applied Science, 1984.

26. Horneck, R.W. : Numerical Methods. Prentice-Hall, New Jersey, 1975.

27. Iskovitz, I.; Chang, T.Y.P.; and Saleeb, A.F.: Finite Element Implementation of State Variable-Based Viscoplastic models. High-Temperature Constitutive Modelling-Theory and Application, A. Freed and K. P. Walker, eds., ASME MD-Vol. 26 \& AMD-Vol. 121, 1991, pp. 307-321.

TABLE I.-FREED-VERRILLI MODEL

CONSTANTS FOR COPPER

[From reference 17.]

\begin{tabular}{|c|l|l|}
\hline Constant & Unit & \multicolumn{1}{|c|}{ Value } \\
\hline$E$ & $\mathrm{MPa}$ & $165000-125 T$ \\
$\alpha$ & ${ }^{\circ} \mathrm{C}^{-1}$ & $15 \times 10^{-6}+5 \times 10^{-9} T$ \\
$\nu$ & - & 0.34 \\
$A$ & $\mathrm{~s}^{-1}$ & $50 \times 106$ \\
$D_{0}$ & $\mathrm{MPa}$ & 1.5 \\
$h$ & $\mathrm{MPa}$ & 500 \\
$H$ & $\mathrm{MPa}$ & 5000 \\
$L$ & $\mathrm{MPa}$ & $25 \exp (-T / 300)$ \\
$n$ & - & 5 \\
$Q$ & $\mathrm{~J} / \mathrm{mole}$ & 200000 \\
$S$ & $\mathrm{MPa}$ & 14.3 \\
$T_{m}$ & $\mathrm{~K}$ & 1356 \\
& & \\
\hline
\end{tabular}


TABLE II.-BODNER-PARTOM MODEL CONSTANTS F0R B 1900+Hf [From reference 18.]

(a) Temperature-independent constants

$$
\begin{aligned}
& m_{1}=0.270 \mathrm{MPa}^{-1} \\
& m_{2}=1.520 \mathrm{MPa}^{-1} \\
& \alpha_{1}=0.0 \\
& Z_{1}=3000 \mathrm{MPa} \\
& Z_{3}=1150 \mathrm{MPa} \\
& r_{1}=r_{2}=2 \\
& D_{0}=1 \times 10^{4} \mathrm{~s}^{-1}
\end{aligned}
$$

(b) Temperature-dependent constants

\begin{tabular}{|l|r|r|r|r|}
\hline \multirow{2}{*}{ Constant } & \multicolumn{4}{|c|}{ Temperature, ${ }^{\circ} \mathrm{C}$} \\
\cline { 2 - 5 } & \multicolumn{1}{|c|}{$\leq 760$} & \multicolumn{1}{c|}{981} & \multicolumn{1}{c|}{1093} \\
\hline$n$ & 1.055 & 1.03 & 0.8500 & 0.7000 \\
$Z_{0}(\mathrm{MPa})$ & 2700 & 2400 & 1900 & 1200 \\
$A_{1}=A_{2}\left(\mathrm{~s}^{-1}\right)$ & 0 & 0.0055 & 0.0200 & 0.2500 \\
$Z_{2}=Z_{0}(\mathrm{MPa})$ & 2700 & 2400 & 1900 & 1200 \\
\hline
\end{tabular}

(c) Elastic constants (MPa; $T$ in ${ }^{\circ} \mathrm{C}$ )

$$
\begin{aligned}
& E=1.987 \times 10^{5}+16.78 T-0.1034 T^{2}+1.143 \times 10^{-5} T^{3} \\
& G=8.650 \times 10^{4}-17.58 T+0.2321 T^{2}-3.464 \times 10^{-5} T^{3}
\end{aligned}
$$

TABLE III.-COEFFICIENTS FOR LOWER RUNGE-KUTTA METHOD OF ORDER ONE (EULER-CAUCHY

OR RK1L METHOD)

\begin{tabular}{|c|l|c|c|}
\hline$\kappa$ & \multicolumn{1}{|c|}{$\alpha_{\kappa}$} & $\begin{array}{c}\beta_{\kappa \lambda} \\
(\lambda=0)\end{array}$ & $c_{\kappa}$ \\
\hline 0 & 0.0 & & 0.0625000 \\
1 & .5333333 & 0.5333333 & .5333333 \\
\hline
\end{tabular}

TABLE IV.-COEFFICIENTS FOR LOWER RUNGE-KUTTA METHOD OF ORDER TWO (RK2L METHOD)

\begin{tabular}{|c|c|c|c|c|}
\hline \multirow{2}{*}{$*$} & \multirow{2}{*}{$\alpha_{\kappa}$} & \multicolumn{2}{|c|}{$\beta_{\kappa \lambda}$} & \multirow{2}{*}{$c_{\kappa}$} \\
\cline { 3 - 4 } & & \multicolumn{2}{|c|}{$\lambda$} & \\
\cline { 3 - 4 } & & 0 & 1 & \\
\hline 0 & 0.0 & 0.0 & - & 0.2401795 \\
1 & .2500000 & .2500000 & - & .0303030 \\
2 & .6750000 & -.2362500 & 0.9112500 & .7295174 \\
\hline
\end{tabular}


TABLE V.-RESULTS FOR FREED-VERRILLI MODEL

(a) Tensile loading

\begin{tabular}{|l|c|c|c|c|c|c|}
\hline Method & Scheme & $\begin{array}{c}\text { Prescribed } \\
\text { upper error in } \\
\text { Runge-Kutta } \\
\text { method, } \\
\text { RTOL }_{u}\end{array}$ & $\begin{array}{c}\text { Prescribed } \\
\text { upper error in } \\
\text { exponential } \\
\text { method, } \\
\text { ETOL }_{u}\end{array}$ & $\begin{array}{c}\text { Iterations } \\
\text { (CPU) time, } \\
\text { s }\end{array}$ & Error \\
\hline RK2 & I & $10^{-3}$ & - & 104091 & 9.871 & $0.460 \times 10^{-3}$ \\
RK1L & I & $10^{-3}$ & - & 104091 & 10.002 & $.460 \times 10^{-3}$ \\
RK2L & I & $10^{-3}$ & - & 104091 & 12.949 & $.460 \times 10^{-3}$ \\
RK2EXP & II & $10^{-7}$ & $5 \times 10^{-6}$ & $40352(3474 \mathrm{a}+36878 \mathrm{~b})$ & 4.638 & $.416 \times 10^{-3}$ \\
\hline
\end{tabular}

(b) Relaxation loading

\begin{tabular}{|l|l|l|l|l|l|c|}
\hline RK2 & I & $10^{-2}$ & - & 10802 & 1.205 & $0.352 \times 10^{-3}$ \\
RK1L & I & $10^{-2}$ & - & 10802 & 1.205 & $.352 \times 10^{-3}$ \\
RK2L & I & $10^{-2}$ & - & 10802 & 1.530 & $.352 \times 10^{-3}$ \\
RK2EXP & II & $10^{-4}$ & $10^{-4}$ & $8381\left(1001 \mathrm{a}+7380^{\mathrm{b}}\right)$ & 1.096 & $.364 \times 10^{-3}$ \\
\hline
\end{tabular}

(c) Cyclic loading

\begin{tabular}{|l|l|l|l|l|l|r|}
\hline RK2 & I & $10^{-2}$ & - & 32105 & 3.508 & $0.227 \times 10^{-3}$ \\
RK1L & I & $10^{-2}$ & - & 32102 & 3.554 & $.223 \times 10^{-3}$ \\
RK2L & I & $10^{-2}$ & - & 32105 & 4.487 & $.223 \times 10^{-3}$ \\
RK2EXP & II & $10^{-6}$ & $10^{-4}$ & $26160\left(11157^{\mathrm{a}}+15003 \mathrm{~b}\right)$ & 3.259 & $.343 \times 10^{-3}$ \\
\hline
\end{tabular}

aNumber of times Runge-Kutta method was used.

bNumber of times exponential method was used. 
TABLE VI.-RESULTS FOR BODNER-PARTOM MODEL

(a) Tensile loading

\begin{tabular}{|l|c|c|c|c|c|c|}
\hline Method & Scheme & $\begin{array}{c}\text { Prescribed } \\
\text { upper error in } \\
\text { Runge-Kutta } \\
\text { method, } \\
\text { RTOL }_{u}\end{array}$ & $\begin{array}{c}\text { Prescribed } \\
\text { upper error in } \\
\text { exponential } \\
\text { method, } \\
\text { ETOL }_{u}\end{array}$ & Iterations & $\begin{array}{c}\text { Execution } \\
\text { (CPU) time, } \\
\text { s }\end{array}$ & Error \\
\hline RK2 & I & $10^{-2}$ & - & 11510 & 1.166 & $0.174 \times 10^{-3}$ \\
RK1L & I & $10^{-2}$ & - & 11510 & 1.183 & $.174 \times 10^{-3}$ \\
RK2L & I & $10^{-2}$ & - & 11510 & 1.620 & $.174 \times 10^{-3}$ \\
RK2EXP & II & $10^{-1}$ & $10^{-1}$ & $2129\left(1948^{2}+181^{\text {b }}\right)$ & .223 & $.104 \times 10^{-3}$ \\
\hline
\end{tabular}

(b) Relaxation loading

\begin{tabular}{|l|l|l|l|l|l|l|}
\hline RK2 & I & $10^{-3}$ & - & 61000 & 6.270 & $0.525 \times 10^{-4}$ \\
RK1L & I & $10^{-3}$ & - & 61000 & 6.299 & $.525 \times 10^{-4}$ \\
RK2L & I & $10^{-3}$ & - & 61000 & 8.712 & $.525 \times 10^{-4}$ \\
RK2EXP & I & $10^{-3}$ & $10^{-3}$ & $61008(50107 \mathrm{a}+10901 \mathrm{~b})$ & 6.653 & $.525 \times 10^{-4}$ \\
\hline
\end{tabular}

(c) Cyclic loading

\begin{tabular}{|l|l|l|l|l|l|l|}
\hline RK2 & I & $10^{-5}$ & - & 96058 & 10.117 & $0.858 \times 10^{-5}$ \\
RK1L & I & $10^{-5}$ & - & 96058 & 10.362 & $.858 \times 10^{-5}$ \\
RK2L & I & $10^{-5}$ & - & 96058 & 13.966 & $.858 \times 10^{-5}$ \\
RK2EXP & II & $10^{-5}$ & $10^{-5}$ & $96059\left(62941^{\mathrm{a}+33} 118^{\mathrm{b}}\right)$ & 10.971 & $.858 \times 10^{-5}$ \\
\hline
\end{tabular}

aNumber of times Runge-Kutta method was used.

bNumber of times exponential method was used. 

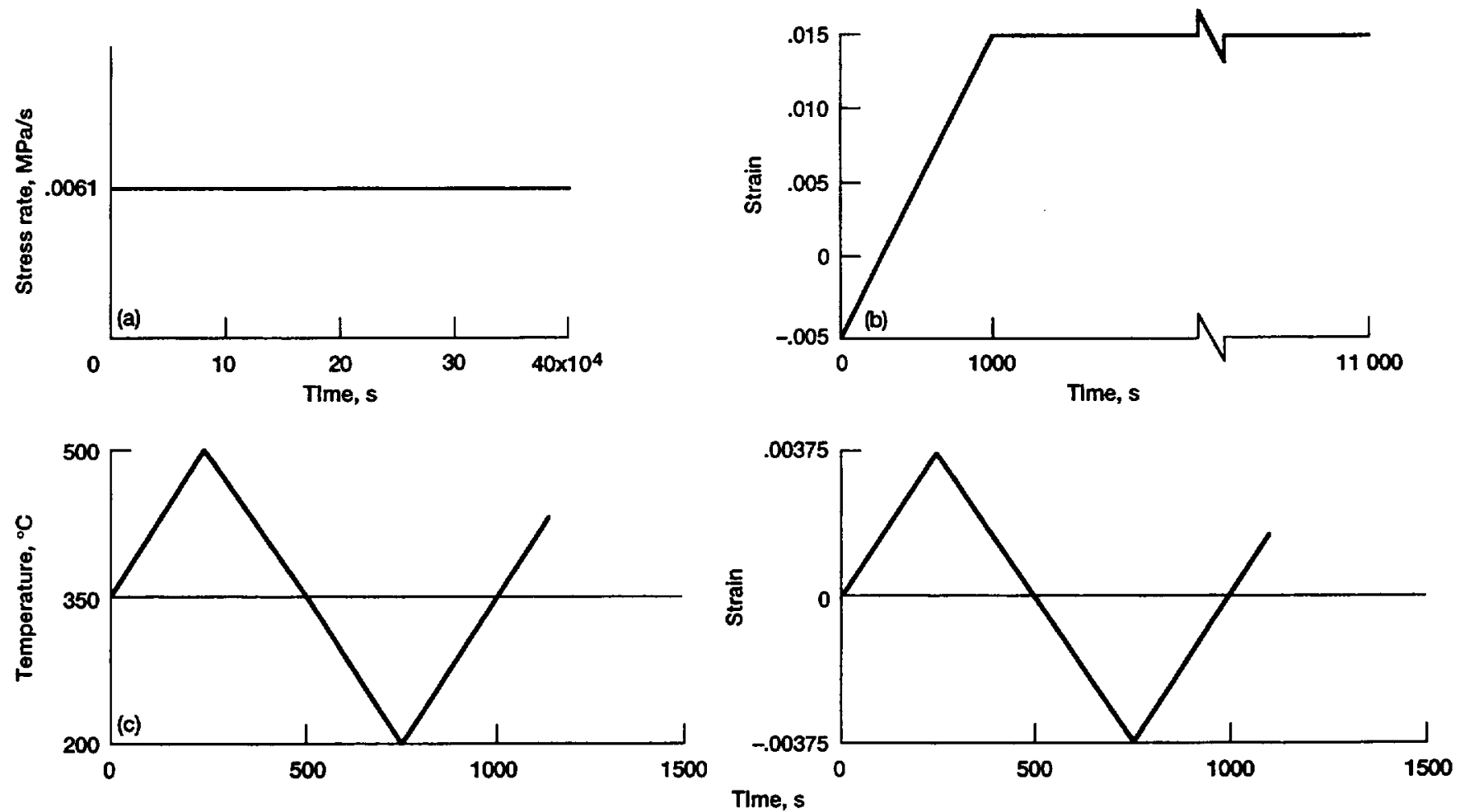

Figure 1.-Thermal/mechanical loadings for Freed-Verrilli model. (a) Tensile loading. Temperature, $149^{\circ} \mathrm{C}$. (b) Relaxation loading. Temperature, $316^{\circ} \mathrm{C}$. (c) Cyclic loading. Temperature, $200 \Leftrightarrow 500^{\circ} \mathrm{C}$. 

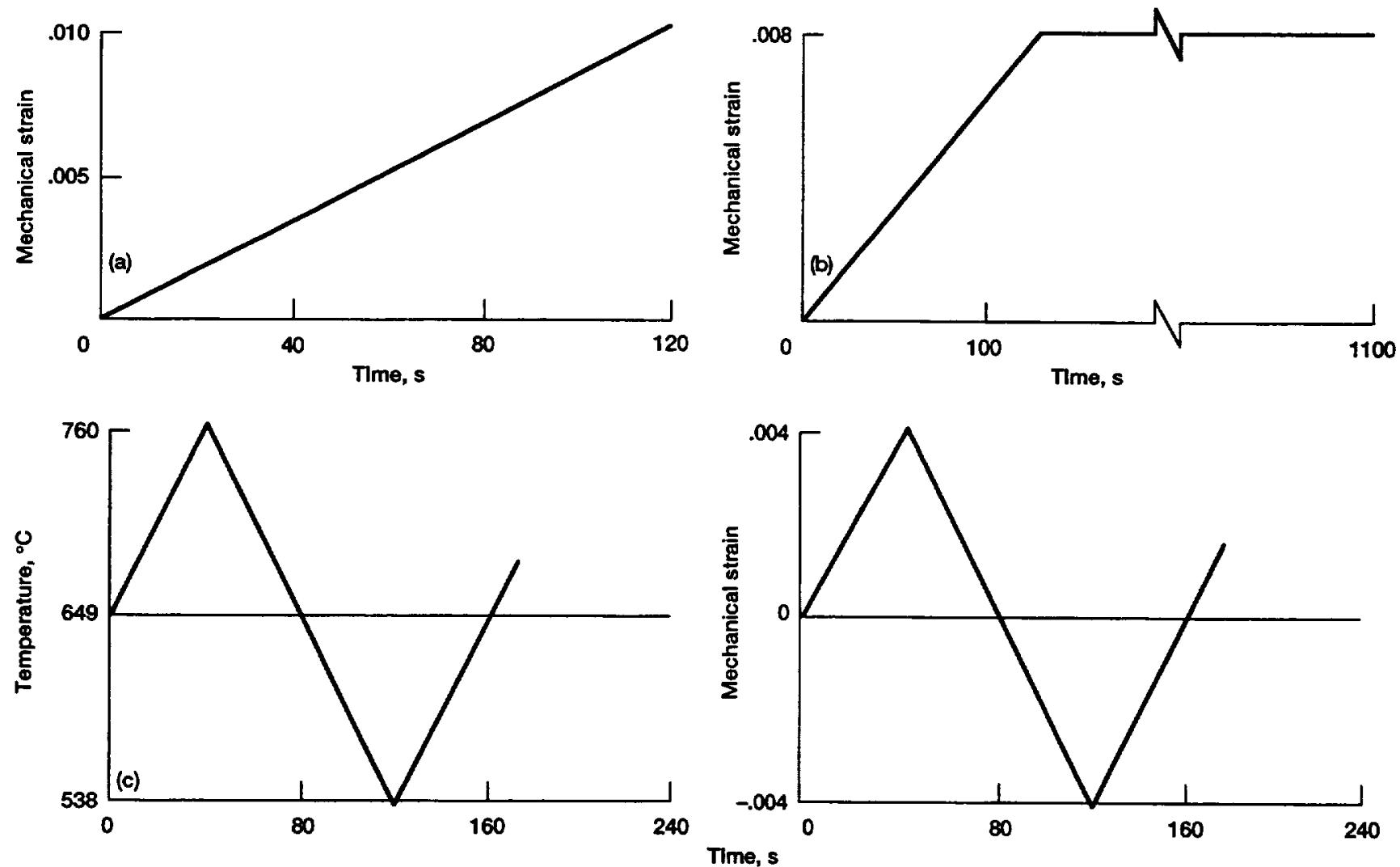

Figure 2-Thermal/mechanical loadings for Bodner-Partom model. (a) Tensile loading. Temperature, $871^{\circ} \mathrm{C}$. (b) Relaxation loading. Temperature, $871^{\circ} \mathrm{C}$. (c) Cyclic loading. Temperature, $538 \Leftrightarrow 760^{\circ} \mathrm{C}$. 

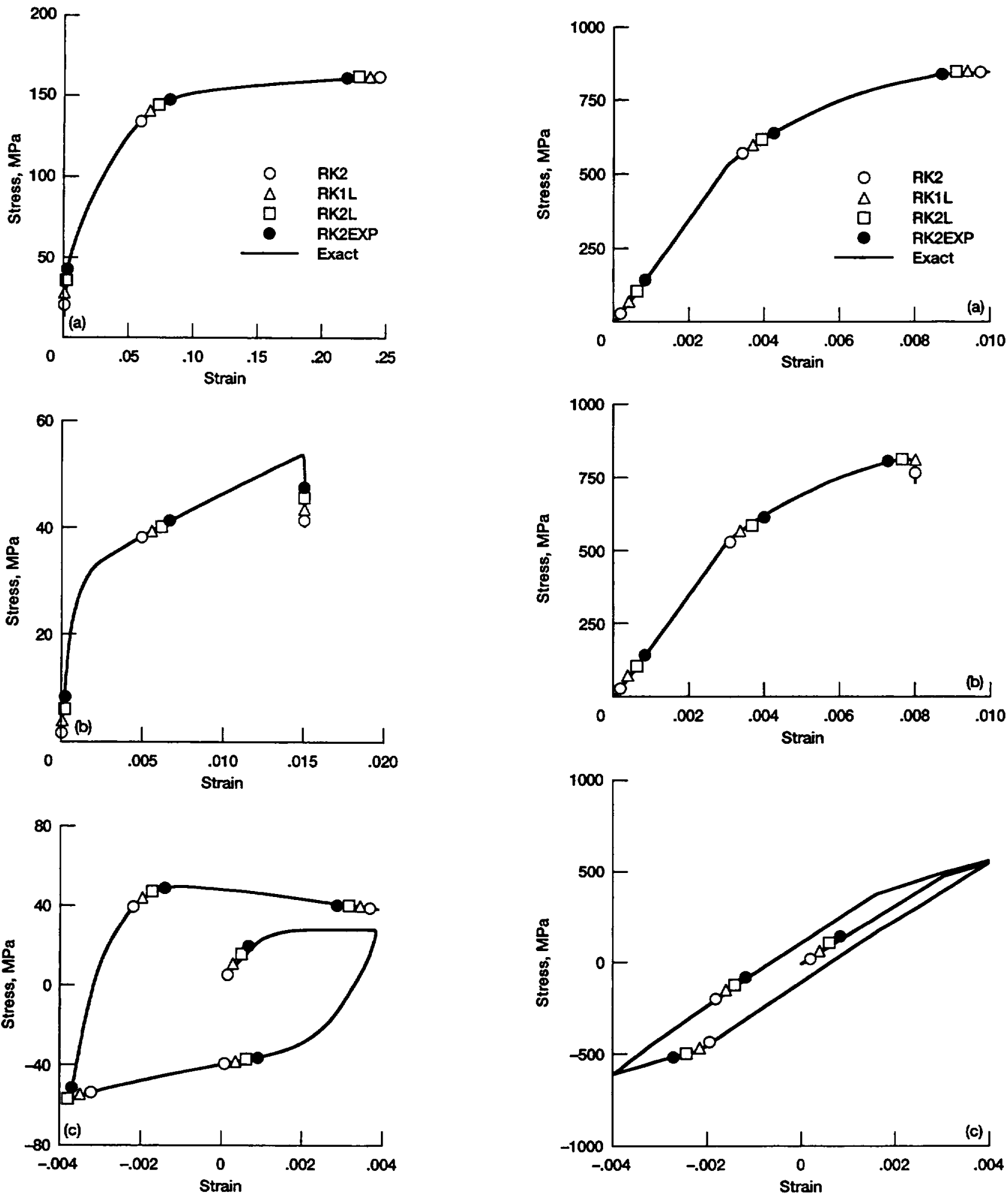

Figure 3.-Stress-strain and relaxation curves and hysteresis loops for Freed-Verrili model (copper).

(a) Stress-strain curve. Stress rate, $6.10 \times 10^{-3} \mathrm{MPa} / \mathrm{s}$; temperature, $149^{\circ} \mathrm{C}$. (b) Relaxation curve. Strain rate, $1.50 \times 10^{-6} / \mathrm{s}$; temperature, $316^{\circ} \mathrm{C}$. (c) Hysteresis loops. Strain rate, $1.50 \times 10^{-5} / \mathrm{s}$; strain range, \pm 0.00375 ; temperature, $200 \Leftrightarrow 500^{\circ} \mathrm{C}$.

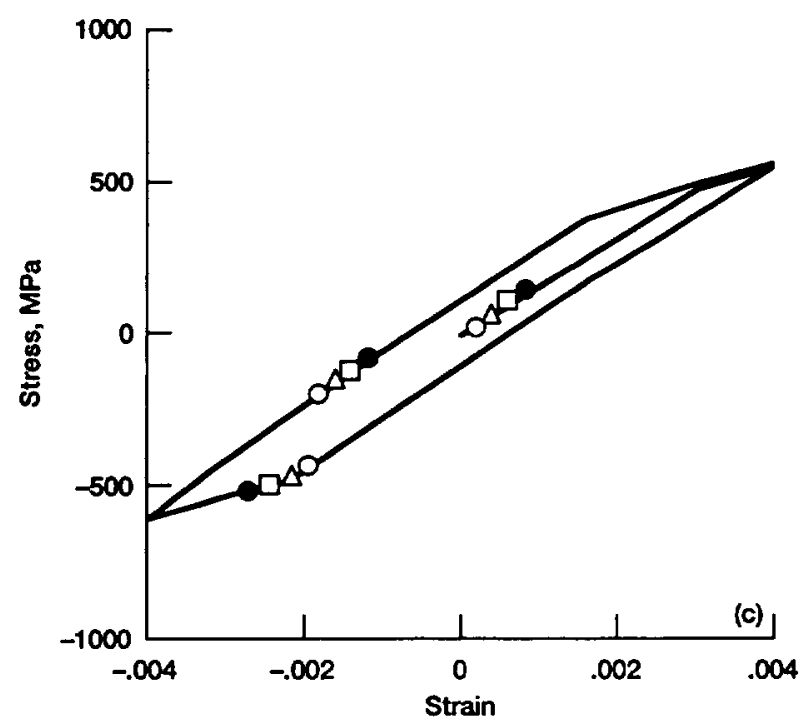

Figure 4.-Stress-strain and relaxation curves and hysteresis loops for Bodner-Partom model $(\mathrm{B1} 1900+\mathrm{Hf})$. (a) Stress-strain curve. Strain rate, $8.333 \times 10^{-6} / \mathrm{s}$; temperature, $871^{\circ} \mathrm{C}$.

(b) Relaxation curve. Strain rate, $8.333 \times 10^{-5} / \mathrm{s}$; maximum strain, 0.008 ; temperature, $871^{\circ} \mathrm{C}$. (c) Hysteresis loops. Strain rate, $1.0 \times 10^{-4} / \mathrm{s}$; strain range, \pm 0.004 ; temperature, $538 \Leftrightarrow$ $760^{\circ} \mathrm{C}$. 
Public reporting burden for this collection of information is estimated to average 1 hour per response, including the time for reviewing instructions, searching existing data sources, gathering and maintaining the data needed, and completing and reviewing the collection of information. Send comments regarding this burden estimate or any other aspect of this collection of information, including suggestions for reducing this burden, to Washington Headquarters Services, Directorate lor Information Operations and Reports. 1215 Jefferson Davis Highway, Suite 1204, Arlington, VA 22202-4302, and to the Office of Managernent and Budget. Paperwork Reduction Project (0704-0188), Washington, DC 20503.

\begin{tabular}{|l|l|l}
\hline 1. AGENCY USE ONLY (Leave blank) & $\begin{array}{c}\text { 2. REPOAT DATE } \\
\text { August } 1994\end{array}$ & $\begin{array}{c}\text { 3. REPORT TYPE AND DATES COVERED } \\
\text { Final Contractor Report }\end{array}$ \\
\hline
\end{tabular}

\section{TITLE AND SUBTITLE}

Efficient and Accurate Explicit Integration Algorithms With Application to Viscoplastic Models

6. AUTHOR(S)

Vinod K. Arya

7. PERFORMING ORGANIZATION NAME(S) AND ADDRESS(ES)

University of Toledo

Toledo, Ohio 43606

9. SPONSORING/MONITORING AGENCY NAME(S) AND ADDRESS(ES)

National Aeronautics and Space Administration

Lewis Research Center

Cleveland, Ohio 44135-3191
5. FUNDING NUMBERS

WU-505-63-5B

NCC3-120

8. PERforming ORGANIZATION REPORT NUMBER

E-8930

10. SPONSORINGMONITORING AGENCY REPORT NUMBER

NASA CR-195342

\section{SUPPLEMENTARY NOTES}

Project Manager, Gary R. Halford, Structures Division, NASA Lewis Research Center, organization code 5200, (216) 433-3265.

12.. DISTRIBUTIONAVAILABILITY STATEMENT 12b. DISTRIBUTION CODE

Unclassified - Unlimited

Subject Categories 39 and 64

13. ABSTRACT (Maximum 200 words)

Several explicit integration algorithms with self-adaptive time integration strategies are developed and investigated for efficiency and accuracy. These algorithms involve the Runge-Kutta second order, the lower Runge-Kutta method of orders one and two, and the exponential integration method. The algorithms are applied to viscoplastic models put forth by Freed and Verrilli and Bodner and Partom for thermal/mechanical loadings (including tensile, relaxation, and cyclic loadings). The large amount of computations performed showed that, for comparable accuracy, the efficiency of an integration algorithm depends significantly on the type of application (loading). However, in general, for the aforementioned loadings and viscoplastic models, the exponential integration algorithm with the proposed self-adaptive time integration strategy worked more (or comparably) efficiently and accurately than the other integration algorithms. Using this strategy for integrating viscoplastic models may lead to considerable saving in computer time (better efficiency) without adversely affecting the accuracy of the results. This conclusion should encourage the utilization of viscoplastic models in the stress analysis and design of structural components.

\begin{tabular}{|c|c|c|}
\hline \multicolumn{3}{|c|}{$\begin{array}{l}\text { 14. SUBJECT TERMS } \\
\text { Integration algorithms; Numerical methods; Viscoplasticity; "Stiff" differential } \\
\text { equations }\end{array}$} \\
\hline $\begin{array}{l}\text { 17. SECURITY CLASSIFICATION } \\
\text { OF REPORT } \\
\text { Unclassified }\end{array}$ & $\begin{array}{l}\text { 18. SECURITY CLASSIFICATION } \\
\text { OF THIS PAGE } \\
\text { Unclassified }\end{array}$ & $\begin{array}{l}\text { 19. SECURITY CLASSIFICATION } \\
\text { OF ABSTRACT } \\
\text { Unclassified }\end{array}$ \\
\hline
\end{tabular}

15. NUMBER OF PAGES 25

16. PRICE CODE

$\mathrm{AO3}$

20. LIMITATION OF ABSTRACT 